\title{
Comparison of NHS and private patients undergoing elective transurethral resection of the prostate for benign prostatic hypertrophy
}

\author{
Nick Black, Mark Pettigrew, Klim McPherson
}

\begin{abstract}
Objectives-To compare the operative thresholds and clinical management of men undergoing elective transurethral resection of the prostate for benign prostatic hypertrophy in the NHS and privately.
\end{abstract}

Design-Cohort study of patients recruited by 25 surgeons during 1988 .

Setting-Hospitals in Oxford and North West Thames regions.

Patients-Of 400 consecutive patients, 129 were excluded because of open surgery (nine), lack of surgeons' information (three), and emergency admission (117) and three failed to give information, leaving 268 patients, 214 NHS patients and 54 private patients.

Main measures-Sociodemographic factors, prevalence and severity of symptoms, comorbidity, general health (Nottingham health profile) obtained from patient questionnaire preoperatively and reasons for operating, and operative management obtained from surgeons perioperatively.

Results-NHS and private patients were similar in severity of symptoms and prevalence of urinary tract abnormalities. They differed in four respects: NHS patients' general health was poorer as a consequence of more comorbid conditions $(49,23 \% \quad v 7,13 \%$ in severe category); the condition had a greater detrimental effect on their lives $(36,17 \%$ $v 2,4 \%$ severely affected; $p<0.01$ ); private patients received more personalised care more quickly and were investigated more before surgery, $(29,54 \% \quad v \quad 60,20 \%$ receiving ultrasonography of the urinary tract); and NHS patients stayed in hospital longer $(57,27 \%$ v 3, 6\% more than seven days; $\mathbf{p}<0.001)$.

Conclusions-Private patients' need for surgery, judged by symptom severity, was as great as that of NHS patients, and there was no evidence of different operative thresholds in the two sectors, but, judged by impact on lifestyle, NHS patients' need was greater.

(Quality in Health Care 1993;2:11-16)

\section{Introduction}

The private sector is making a large and increasing contribution to the provision of elective surgery in the United Kingdom, particularly in south eastern England. ${ }^{1-3}$ Attitudes to this development vary; some see the private sector as providing much needed help in relieving need that cannot be met by the NHS and others believe that private provision is largely a non-essential luxury for a privileged few.

Several hypotheses concerning differences between the two sectors have been suggested. Private patients have less severe symptoms but higher expectations and are therefore bothered more by their condition; they undergo more extensive preoperative investigation, partly because of financial incentives created by a fee for service method of payment and partly because of stricter budgetary control in the NHS; as a result, private patients are, for example, more likely to have a diagnosis of chronic urinary retention because of an apparently higher prevalence of pathological changes; and they wait less time for elective surgery and are more likely to be operated on by a consultant surgeon.

Only one study has attempted to test some of these hypotheses. ${ }^{4}$ Comparison of private and NHS patients undergoing cholecystectomy showed that they were similar in age, sex, indications for surgery, and comorbidity. The only differences were that NHS patients were more likely to have been admitted as emergency cases and to have undergone more than one ultrasound investigation. Being based on retrospective data obtained from case notes, that study could investigate only a limited number of factors. In contrast, this paper presents data collected prospectively as part of a study of the indications for transurethral resection of the prostate for benign prostatic hypertrophy and its outcome. ${ }^{5}$

\section{Patients and methods}

During 1988, 25 surgeons in the Oxford and North West Thames health regions recruited between 10 and 40 men (depending on their annual volume of surgery) admitted for transurethral resection for benign prostatic hypertrophy. Men who were known to have a malignant neoplasm of the prostate, a first language other than English, or who were permanently resident outside the United Kingdom were excluded. The surgeons recruited around $80 \%$ of eligible NHS patients and $55 \%$ of eligible private patients treated during the recruitment period. 
Four hundred patients were invited to join the study; 397 (nine of whom were subsequently excluded as they underwent open surgery and constituted too small a group for comparison) completed a questionnaire in hospital while awaiting their operation. We failed to obtain clinical information from the surgeons of three of the patients, leaving 385 patients in the study, 326 treated in the NHS and 59 treated privately. Of these 385,117 patients presented as emergency cases with a history of acute urinary retention and were excluded as private hospitals are not equipped to admit such cases. This left 214 NHS and 54 private patients admitted as non-emergency elective cases.

The preoperative questionnaire elicited information regarding socioeconomic background, preoperative health status, and the incidence of urinary symptoms over the past month (hesitancy, poor stream, postmicturition dribbling, fullness, frequency, and dysuria) on a five point scale ranging from "never" to "always." Nocturia was recorded as the number of times the patients needed to get up during the night to urinate (never/once 0, twice 1 , three times 2 , four times 3 , five times or more 4 ). The scores for each of the seven urinary symptoms were summed (never 0 , a few times 1 , half the time 2 , most of the time 3 , always 4) to give an "all symptoms index" ranging from 0-28 (none 0 , mild 1-7, moderate 8-14, severe 15-28). A lifestyle effect index was constructed to reflect the effect of the symptoms on lifestyle (refraining from activity because of urgency or incontinence ("wetting"), being embarrassed about frequency or hesitancy, and worrying about acute retention) ranging from 0-20 (none 0 , mild 1-5, moderate 6-10, severe 11-20).

Perceived health status was assessed using the first part of the Nottingham health profile, a two part questionnaire which takes about 10 minutes to complete. ${ }^{6}$ The first part consists of 38 statements covering six areas of health: energy level, experience of pain, emotional reactions, sleep, social isolation, and physical mobility. The respondent is required to affirm or refute each individual statement depending on whether the item applies to himself "in general." The responses to part I of the profile were weighted for perceived severity as described elsewhere ${ }^{7}$ so that the total weighted score for each section ranged from zero (every statement refuted) to 100 (every statement answered affirmatively). In view of the sensitivity of the profile to age, sex, and social class, comparisons between NHS and private patients were adjusted to control for the significant differences in social class observed between the two groups. Controlling for sex was clearly irrelevant, and the range of ages was constrained, making adjustment unnecessary.

The surgeons were asked to complete a questionnaire perioperatively for each patient, which included preoperative assessment (symptoms, reason for operating, and comorbidity), use of investigations (biochemical and haematological investigations, urine culture, urodynamics, electrocardiography, and imaging), and operative management (grade of surgeon, length of operation, amount of tissue resected, blood transfusions, and use of postoperative catheter). Comorbidity was assessed by means of closed questions about a history of myocardial infarction, stroke, cardiac insufficiency, malignancies, diabetes, and hypertension and by open questions about other current illnesses, treatments, hospital admissions in the past year, and previous surgery. Responses to open questions were categorised (none 0 , mild 1 , moderate 2, severe 3 ). An overall comorbidity index was constructed by summing the patients' responses to all questions about current conditions and categorising the total (none 0, mild 1 , moderate $2-4$, severe 5 or more)

$\chi^{2}$ and Fisher's exact tests (when appropriate) were used to compare proportions when variables were measured on an ordinal scale. For analysis of group differences with the Nottingham health profile analysis of variance was used, with social class as a covariate.

\section{Results}

SOCIODEMOGRAPHIC CHARACTERISTICS

Private patients were younger than NHS patients (mean age $66 \cdot 8(\mathrm{SEM} \mathrm{1.2)}$ ) years $v$ $69.9(0.6)$ years respectively; $p<0.02)$, more likely to have continued in full time education after the age of $19(20,37 \%$ v $19,9 \%$; $\mathrm{p}<0.0001)$, to belong to the professional and managerial social classes $(43,80 \% v 79,37 \%$; $\mathrm{p}<0.0001)$, and to own their own house (50, $93 \%$ v $141,66 \% ; \mathrm{p}<0.001)$. Private and NHS patients were similar in their marital status, whether they lived alone or not, and the amount of paid domestic help they received.

\section{GENITOURINARY SYMPTOMS}

Private patients tended to report less severe symptoms than NHS patients, but the differences were not significant (none/mild 1, $2 \%$ v $21,10 \%$; moderate $33,61 \%$ v $96,45 \%$; severe $20,37 \% \vee 97,45 \%$ for private and NHS patients respectively). However, when the impact of symptoms on lifestyle was considered, the difference became significant: $36(17 \%)$ of NHS patients refrained from some activity most or all of the time because of worrying about needing a toilet compared with $1(2 \%)$ of private patients $(\mathrm{p}<0.02)$, and $32(15 \%)$ of them refrained from activities because of anxiety about wetting themselves in public compared with none of the private patients $(p<0.01)$. These differences were reflected in the overall measure of the impact of symptoms - that is, the lifestyle effect index - in which $36(17 \%)$ of NHS patients were categorised as severely affected compared with $2(4 \%)$ of private patients $(p<0 \cdot 01)$.

Whether the cause was benign prostatic hypertrophy or not, NHS patients were less likely than private patients to be able to achieve an erection when sexually stimulated $(122,57 \% \vee 15,28 \% ; \mathrm{p}<0.01)$, a difference that persisted after adjusting for age. NHS patients were also less likely to wake up with 
an erection $(83,39 \% v 12,22 \%)$ and more likely to report that their sex life had been spoilt by their prostate problem $(51,24 \%, v 6$, $11 \%$ ), though these differences did not reach significance.

COMORBIDITY

The two groups of patients were not significantly different in terms of their histories of myocardial infarction, stroke, cardiac

Table 1 History of comorbidity as reported by surgeons and patients. Figures are numbers (percentages) of patients

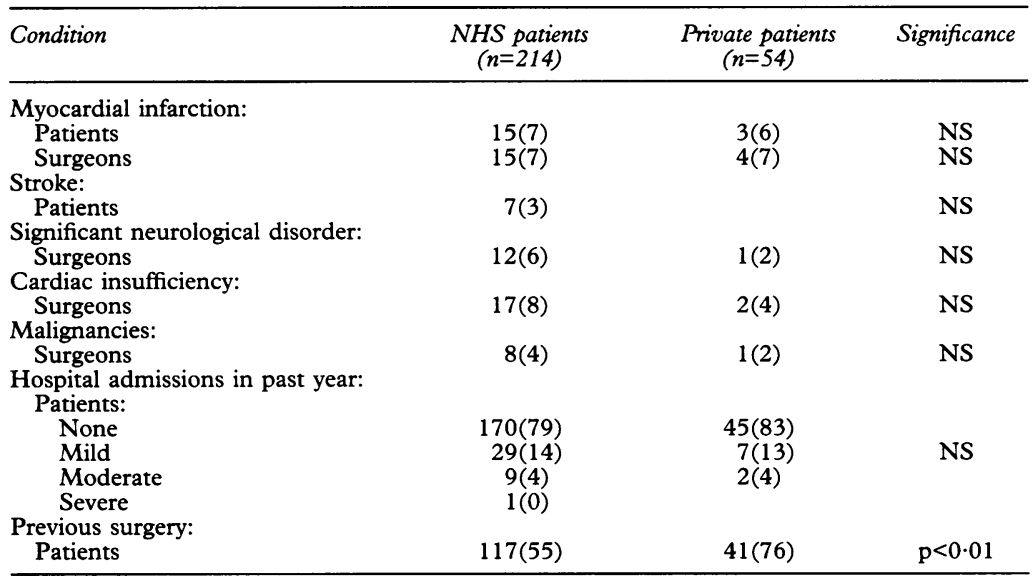

Table 2 Types of surgery previously undergone. Figures are numbers (percentages) of patients

\begin{tabular}{lcc}
\hline Category & $\begin{array}{c}\text { NHS patients } \\
(n=214)\end{array}$ & $\begin{array}{c}\text { Private patients } \\
(n=54)\end{array}$ \\
\hline Cardiovascular & $9(4)$ & $1(2)$ \\
Genitourinary & $17(8)$ & $6(11)$ \\
Ear, nose, and throat & $21(10)$ & $17(31)$ \\
Orthopaedic & $26(12)$ & $10(19)$ \\
Abdominal & $40(19)$ & $22(41)$ \\
Hernia repair & $46(22)$ & $6(11)$ \\
Rectal or anal & $16(7)$ & $10(19)$ \\
Varicose veins & $7(3)$ & $1(2)$ \\
Skin & $2(1)$ & $4(7)$ \\
Thyroid & $3(1)$ & \\
Ophthalmological & $13(6)$ & $1(2)$ \\
Other minor & $9(4)$ & $7(13)$ \\
\hline
\end{tabular}

Table 3 Current comorbidity as reported by surgeons and patients. Figures are numbers (percentages) of patients

\begin{tabular}{|c|c|c|c|}
\hline & $\begin{array}{c}\text { NHS patients } \\
(n=214)\end{array}$ & $\begin{array}{l}\text { Private patients } \\
\quad(n=54)\end{array}$ & Significance \\
\hline \multicolumn{4}{|l|}{ Respiratory problems: } \\
\hline \multicolumn{4}{|l|}{ Hypertension: } \\
\hline Patients & $49(23)$ & $7(13)$ & NS \\
\hline Surgeons & $65(30)$ & $13(24)$ & NS \\
\hline \multicolumn{4}{|l|}{ Diabetes: } \\
\hline Surgeons & $3(1)$ & $2(4)$ & NS \\
\hline \multicolumn{4}{|l|}{ Other conditions: } \\
\hline \multicolumn{4}{|l|}{ Patients: } \\
\hline None & $135(63)$ & $29(54)$ & \\
\hline Mild & $42(20)$ & $13(24)$ & NS \\
\hline Moderate & $27(13)$ & $10(19)$ & \\
\hline Severe & $10(5)$ & $2(4)$ & \\
\hline \multicolumn{4}{|l|}{ Current treatment: } \\
\hline \multicolumn{4}{|l|}{ Patients: } \\
\hline None & $102(48)$ & $30(56)$ & \\
\hline For mild condition & $17(8)$ & $6(11)$ & NS \\
\hline For moderate condition & $70(33)$ & $15(28)$ & \\
\hline For severe condition & $25(12)$ & $3(6)$ & \\
\hline \multicolumn{4}{|l|}{ Surgeons: } \\
\hline None & $98(46)$ & $38(70)$ & \\
\hline For mild condition & $17(8)$ & $6(11)$ & $\mathrm{p}<0.005$ \\
\hline For moderate condition & $57(27)$ & $8(15)$ & \\
\hline For severe condition & $42(20)$ & $2(4)$ & \\
\hline \multirow{2}{*}{\multicolumn{4}{|c|}{$\begin{array}{l}\text { Comorbidity index: } \\
\text { Patients: }\end{array}$}} \\
\hline & & & \\
\hline None & $67(32)$ & $15(28)$ & \\
\hline Mild & $17(8)$ & $10(19)$ & NS \\
\hline Moderate & $75(35)$ & $22(41)$ & \\
\hline Severe & $49(23)$ & $7(13)$ & \\
\hline
\end{tabular}

insufficiency, and malignancies (table 1). The incidence and severity of hospital admissions during the previous 12 months were similar. The only feature of their histories that differed was that private patients were more likely to have undergone surgery $(76 \%$ v $55 \%$; $\mathrm{p}<0 \cdot 01$ ), mainly because of a higher incidence of abdominal surgery (of the biliary tract, stomach, appendix, and colon), rectal or anal surgery, and ear, nose, and throat surgery among private patients (table 2).

For current conditions the prevalence of hypertension, diabetes, and other conditions were similar in the two groups (table 3). However, NHS patients were more likely than private patients to be reported by their surgeons as having significant respiratory problems $(10 \% v 0 \% ; \mathrm{p}<0.05)$ and to be regularly receiving treatment $(116,54 \% v 16$, $30 \%$; $<<0.005$ ), which were more likely to be for a moderate or severe condition rather than a mild condition (99, 46\% $v 10,19 \%$; $\mathrm{p}<0.05)$. Differences in the reported use of treatments probably arose through surgeons underrecording in the case of private patients as no significant differences were reported by the patients themselves.

The overall comorbidity index, reflecting both past and current problems, disclosed a higher proportion of NHS patients in the severe category $(23 \% v 13 \%)$, though this was not significant.

\section{GENERAL HEALTH STATUS}

Private patients were less likely to rate their general health over the previous month as only fair or poor $(19 \%)$ compared with NHS patients $(36 \%)$ (table 4$)$. The Nottingham health profile disclosed similar significant differences in all dimensions apart from that of energy level. These differnces ceased to be significant once the social class composition of the two groups was controlled for.

When asked to compare their general health with that of others NHS patients did not perceive themselves as being significantly worse off. This may reflect that respondents compared themselves with men of similar socioeconomic status: NHS patients tended to compare themselves with men of lower socioeconomic status (and thus poorer general health) than did private patients, therefore their expectations were not as high as those of private patients.

INVESTIGATIONS PERFORMED

Similar proportions of private and NHS patients underwent the commonest investigations used: measurements of haemoglobin $(52,96 \% \vee 206,96 \%)$ and blood urea concentrations $(37,69 \% v 166,78 \%)$, urine culture $(48,89 \% v 177,83 \%)$, radiology of the urinary tract $(21,39 \%$ v $81,38 \%)$, determination of urinary flow rate $(18,33 \% v$ $63,29 \%)$, and cystometry ( $3,6 \% v 8,4 \%)$. In contrast, surgeons were less likely to report having measured serum creatinine concentration in private patients $(27,50 \% v 167,78 \%$ $\mathrm{p}<0.0001)$ and performed electrocardiography $(15,28 \%, v 192,90 \% ; \mathrm{p}<0.0001)$; the last 
Table 4 Comparison of general health status before surgery. Figures are numbers (percentages) of patients, unless otherwise stated

\begin{tabular}{|c|c|c|c|}
\hline & $\begin{array}{l}\text { NHS patients } \\
\quad(n=214)\end{array}$ & $\begin{array}{l}\text { Private patients } \\
\quad(n=54)\end{array}$ & Significance \\
\hline \multicolumn{4}{|l|}{$\begin{array}{l}\text { Self rating of general health } \\
\text { over previous month: }\end{array}$} \\
\hline Poor or fair & $76(36)$ & $10(19)$ & \multirow{3}{*}{$\mathrm{p}<0.05$} \\
\hline Good & $59(28)$ & $20(37)$ & \\
\hline Very good or excellent & $79(37)$ & $24(44)$ & \\
\hline \multicolumn{4}{|l|}{$\begin{array}{l}\text { Nottingham health profile } \\
\text { (mean weighted scores): }\end{array}$} \\
\hline Energy & $24 \cdot 4$ & $22 \cdot 2$ & NS \\
\hline Pain & $9 \cdot 3$ & $4 \cdot 8$ & $p<0.05$ \\
\hline Emotional reactions & $16 \cdot 0$ & $10 \cdot 2$ & $\mathrm{p}<0.05$ \\
\hline Sleep & $27 \cdot 5$ & $18 \cdot 4$ & $\mathrm{p}<0.05$ \\
\hline Social isolation & $9 \cdot 4$ & $2 \cdot 4$ & $\mathrm{p}<0.001$ \\
\hline Physical mobility & $9 \cdot 8$ & $4 \cdot 1$ & $\mathrm{p}<0.001$ \\
\hline \multicolumn{4}{|c|}{$\begin{array}{l}\text { Compared to other men of my age, } \\
\text { my health is: }\end{array}$} \\
\hline Worse than average & $9(4)$ & $1(2)$ & \multirow{3}{*}{ NS } \\
\hline Average & $97(45)$ & $20(37)$ & \\
\hline Better than average & $102(48)$ & $33(61)$ & \\
\hline
\end{tabular}

finding is presumably due to underreporting by the surgeons.

Although similar proportions of patients in the two groups were investigated with radiology of the urinary tract, private patients were more likely to be investigated by ultrasonography $(29,54 \% \quad v \quad 60,28 \%$; $\mathrm{p}<0.001)$. Overall, $81(38 \%)$ of the NHS patients had no imaging performed compared with nine $(17 \%)$ private patients.

RESULTS OF INVESTIGATION

As some investigations were neither performed on all patients nor used at the same rate in both groups, abnormal results need to be considered both as a proportion of patients tested and a proportion of all patients (table 5). The proportions of private and NHS patients with abnormal results were mostly similar, exceptions being a higher proportion of NHS patients with blood pressure $>180 / 90$ $\mathrm{mm} \mathrm{Hg}(18 \% \quad v \quad 4 \% ; \mathrm{p}<0.01)$ and with abnormalities on electrocardiography $(21 \% v$ $9 \% ; \mathrm{p}<0.05)$. These differences in cardiovascular health were reflected in the proportions of patients classified as constituting a moderate or severe anaesthetic risk $(60,28 \%$ of NHS patients $v 5,9 \%$ private patients; $p<0.005)$. Despite the greater use of imaging in private patients the prevalence of abnormalities of the urinary tract was similar to that in NHS patients.
DECISION TO OPERATE

Surgeons' diagnoses were similar for private and NHS patients. Similar proportions of each group were reported as having obstructive symptoms, irritative symptoms, impaired renal function, and a stone or diverticulum in the bladder. However, private patients were more likely to have a diagnosis of recurrent urinary tract infections $(10,19 \% v 4,2 \% ; \mathrm{p}<0.0001)$ and a large residue after voiding $(29,54 \% v$ $71,33 \% ; \mathrm{p}<0.005)$.

Surgeons' prognoses for both types of patients did not differ significantly: imminent renal impairment $(5,12 \% \quad v \quad 45,30 \%)$; imminent bladder damage $(11,85 \% v 45$, $80 \%)$; an episode of acute retention $(25,52 \%$ $v 97,48 \%$ ); recurrent urinary tract infections $(22,47 \% \quad v 78,40 \%)$; and a reduction in quality of life $(4,80 \% v 12,80 \%)$.

Many more NHS than private patients thought their surgeon had no discretion about performing an operation or not $(150,70 \% v$ $23,43 \% ; \mathrm{p}<0.0001)$. The two groups were little different in their views about their prognosis without surgery, though NHS patients were more likely to think they would live as long $(54,25 \% v 6,11 \% ; \mathrm{p}<0.05)$. The level of anxiety about undergoing the operation and their expectation of resulting benefits were similar between the two groups.

OPERATIVE MANAGEMENT

NHS patients had to wait significantly longer than private patients for their operation (mean time 186(19) days $v$ 49(13) days; $\mathrm{p}<0 \cdot 0001$ ). In view of their greater anaesthetic risk, NHS patients were more likely to have a spinal anaesthetic $(65,31 \% v 6,11 \% ; \mathrm{p}<0.01)$. Apart from the greater likelihood of the operation being performed by a consultant surgeon in the private sector $(54,100 \% v 150$, $70 \% ; \mathrm{p}<0.0001)$, there were no other significant differences regarding the procedure (duration, amount of prostatic tissue resected, additional procedures performed, transfusions, and prophylactic antibiotics).

After operation NHS patients were more likely to require a urethral catheter for five days or more $(37,17 \% v 3,6 \% ; \mathrm{p}<0.05)$ and to stay in hospital for more than 7 days (57, $27 \%$ v $3,6 \% ; \mathrm{p}<0.0001)$. However, the

Table 5 Abnormal test results. Figures are numbers (percentages) of patients

\begin{tabular}{lcccc}
\hline Abnormal finding & NHS patients & Private patients & NHS patients $\dagger$ & Private patients $\dagger$ \\
\hline Low haemoglobin concentration & $18 / 214(8)$ & $3 / 54(6)$ & $18 / 206(9)$ & $3 / 52(6)$ \\
Blood pressure (mm Hg): & $62 / 214(29)$ & $10 / 54(19)$ & $62 / 207(30)$ & $10 / 50(20)$ \\
$>160 / 90$ & $37 / 214(17)$ & $2 / 54(4) \star \star$ & $37 / 207(18)$ & $2 / 50(4) \star \star$ \\
> 180/90 & $16 / 214(7)$ & $4 / 54(7)$ & $16 / 177(9)$ & $4 / 48(8)$ \\
Urinary tract infection & $10 / 214(5)$ & $3 / 54(6)$ & $10 / 81(12)$ & $3 / 21(14)$ \\
Radiological findings: & $30 / 214(14)$ & $5 / 54(9)$ & $30 / 81(37)$ & $5 / 21(24)$ \\
$\quad$ Upper urinary tract dilatation & $5 / 214(2)$ & $1 / 54(2)$ & $5 / 81(6)$ & $1 / 21(5)$ \\
Large residue after voiding & $13 / 214(6)$ & $2 / 54(4)$ & $13 / 60(22)$ & $2 / 29(7)$ \\
Diverticula & $28 / 214(13)$ & $9 / 54(17)$ & $28 / 60(47)$ & $9 / 29(31)$ \\
Ultrasonographic findings: & $4 / 214(2)$ & $3 / 54(6)$ & $4 / 60(7)$ & $3 / 29(10)$ \\
Upper urinary tract dilatation & $44 / 214(21)$ & $5 / 54(9) \ddagger$ & $44 / 192(23)$ & $5 / 15(33)$ \\
Large residue after voiding & & &
\end{tabular}

« Percentages of total patients.

†Percentages of patients tested.

$\star \star \star p<0.01$

$\neq \mathrm{p}<0.05$. 
incidence of immediate complications was similar in the two groups (urinary tract infections $23,11 \% v 4,7 \%$; clot retention 17 , $8 \% v 5,9 \%$ ), as was the prevalence of malignancy in the prostatic tissue resected.

\section{Discussion}

Private and NHS patients are similar in the severity of their symptoms and the prevalence of pathological consequences of chronic urine retention. However, they differ in four important respects: the general health of NHS patients is poorer as a consequence of more comorbid conditions; the detrimental effect of benign prostatic hypertrophy on lifestyle is greater for NHS patients than for private patients; private patients receive more personalised care and are investigated more before surgery; and NHS patients stay in hospital for longer.

Before considering these differences in greater detail three methodological issues need to be addressed. The first is selection bias in recruiting patients to the study. The NHS patients who were recruited were slightly younger than would be expected. The lack of any routine data on private patients in the United Kingdom means that the extent of any bias in this sector is uncertain. Discussions with the participating surgeons suggested that failure to recruit primarily resulted from administrative oversight rather than any systematic attempt to select. However, our sample may have been more sick or more healthy than typical private patients. The second issue is that the power of this study to detect significant differences was limited by the sample sizes, and some clinically important differences between the NHS and private patients may have been missed. This underlines the need for further, larger studies comparing the two sectors. The third issue concerns the assumption we made that the two groups of patients would be representative of the operative threshold that exists in the two sectors. Possibly in the NHS, because of an inadequate supply of services, surgeons can admit only the most severely affected patients. If this were the case then the true operative threshold would be lower than that represented by our sample. We do not believe that this fear is justified as the NHS patients included men with a wide range of disease severity, including $10 \%$ with no symptoms or only mild symptoms.

The first main difference between the two patient groups is that the NHS patients have poorer health as a result of other conditions. Before surgery they are more likely to have signs of cardiovascular disease; to be judged a higher anaesthetic risk; to self rate their own health as poor; to report more problems in all but one dimension of the Nottingham health profile; and to suffer from erectile impotence. Given the sociodemographic differences between the groups, this is not a surprising finding. Less obvious is the explanation for the second observed difference between the groups - that is, why benign prostatic hypertrophy seemed to have a more detrimental effect on the lives of NHS patients.

There are three possible explanations. The first is responder bias: NHS patients may have felt the need to report their condition as having a greater impact on their lives because, unlike private patients, they had to convince their surgeon of their need for surgery. If this were true we would have expected the same to occur with the reporting of severity of symptoms and that did not happen. The second explanation concerns the duration of the condition. NHS patients may have had benign prostatic hypertrophy longer than private patients, partly because both general practitioners and surgeons would tend to refer and treat private patients more rapidly. Given the difficulties of obtaining accurate data on the duration of symptoms, we did not attempt to collect this information. The final possible explanation is that NHS patients are more vulnerable as a result of their poorer general health, and the additional burden of benign prostatic hypertrophy has a greater impact on their lifestyle. This would explain why the significant differences in the scores for the Nottingham health profile between the two groups are resolved after adjustment for social class. In other words, much of the difference in health status is due to underlying, coexisting medical and socioeconomic conditions rather than the prostate problem. Though poor socioeconomic conditions are associated with poorer health, they might also be operating directly. Good social conditions can minimise the impact of benign prostatic hypertrophy whereas poor conditions may mean that even mild symptoms may have a significant impact on lifestyle. For these reasons NHS patients would experience a more greatly reduced quality of life than private patients.

The third observed difference was that private patients receive more care faster, waiting a shorter time before surgery, having their upper urinary tract imaged, and having a consultant surgeon to perform the operation. Greater access to services by private patients is also evident in their history of surgery. Whether or not these differences confer any advantage, beyond private patients not having to wait as long for surgery, is not clear. For example, in this study the greater use of imaging investigations of the urinary tract did not disclose a higher prevalence of abnormalities in private than NHS patients. Whether or not these results can be generalised to patients with other conditions is not clear and must await the results of further comparative studies.

The final difference in clinical management was the longer postoperative stay for NHS patients associated with a higher proportion of them having a urethral catheter for five days or more. This difference may reflect the worse general health of NHS patients. Longer postoperative stay may also reflect poorer home conditions or, alternatively, may be due to NHS hospitals being less efficient. Determining which explanation is correct is not possible in this study. 
Finally, is there any difference in the need for surgery between the two groups? This study suggests that the prevalence of urinary tract disease and the severity of prostatic symptoms are similar. However, it may be more important to consider the impact of symptoms on a patient's lifestyle as a measure of need. NHS patients experienced more restrictions in their everyday activities than private patients and on this basis are in greater need.

We thank Maria Ginzler, Jane Davies, and Helen Doll for their help in collecting and preparing the data; Grant Williams an Joe Smith for clinical advice; Ann Barry Flood and two anonymous referees for comments on the paper; the surgeon and patients who participated in the study; Jim McEwan for granting permission to use the Nottingham health profile; and the health services research committee of the Medical Research Council for funding the study.

1 Nicholl JP, Thomas KJ, Williams BT, Knoweldon J Contribution of the private sector to elective surgery in England and Wales. Lancet 1984;ii:89-92.

2 Nicholl JP, Beeby NR, Williams BT. Role of the private sector in elective surgery in England and Wales, 1986. $B M \mathcal{F}$ 1989;292:243-7.

3 McPherson K, Coulter A, Stratton I. Increasing use of private practice by patients in Oxford requiring common elective surgical operations. BMF 1985;291:797-9.

4 Scott EA, Black NA. Appropriateness of cholecystectomy: the public and private sectors compared. Ann Coll Surg the public and private sectors

5 Doll HA, Black NA, McPherson CK, Flood AB, Williams GB, Smith JC. Mortality, morbidity, and complications following TURP for benign prostatic hypertrophy. $\mathcal{F}$ Uro 1992;147:1566-73

6 Hunt SM, McEwan J. The development of a subjective health indicator. Sociology of Health and Ilnes 1980;2:231-46.

7 Hunt SM, McKenna SP, McEwan J, et al. Perceived health age and sex comparisons in a community. $f$ Epidemio Community Health 1984; 34: 281-6. 